\title{
LINEARIZED MULTIPOLE SOLUTIONS AND THEIR REPRESENTATION
}

\author{
J.L. Hernández-Pastora* \\ Departamento de Matemática Aplicada and IUFFyM ${ }^{\dagger}$ \\ Universidad de Salamanca. Salamanca, Spain
}

July 9, 2018

\begin{abstract}
The monopole solution of the Einstein vacuum field equations (Schwarzschild's solution) in Weyl coordinates involves a metric function that can be interpreted as the gravitational potential of a bar of length $2 m$ with constant linear density. The question addressed in this work is whether similar representations can be constructed for Weyl solutions other than the spherically symmetric one.

A new family of static solutions of the axisymmetric vacuum field equations generalizing the $\mathrm{M}-\mathrm{Q}^{(1)}$ solution [7] is developed. These represent slight deviations from spherical symmetry in terms of the relativistic multipole moments (RMM) we wish the solution to contain. A Newtonian object referred to as a dumbbell can be used to describe these solutions in a simple form by means of the density of this object, since the physical properties of the relativistic solution are characterized by its behaviour. The density profile of the dumbbell, which is given in terms of the RMM of the solution, allows us to distinguish general multipole Weyl solutions from the constant-density Schwarzschild solution. The range of values of the multipole moments that generate positive-definite density profiles are also calculated. The bounds on the multipole moments that arise from this density condition are identical to those required for a well-behaved infinite-redshift surface $g_{00}=0$.
\end{abstract}

PACS numbers: 04.20.Cv, 04.20.-q, 04.20.Jb.

*ETS Ingeniería Industrial de Béjar. e-mail address: jlhp@usal.es

†Instituto Universitario de Física Fundamental y Matemáticas. 


\section{Introduction}

As is well known, all the static solutions, with good asymptotical behaviour, of the axially symmetric Einstein vacuum equations are given by the Weyl family of solutions [1]. Each solution of this family is characterized by means of a particular set of coefficients $\left\{a_{n}\right\}$ through a series that provides the metric function of the solution in Weyl coordinates $\{r, \theta\}$ ( $P_{l}$ being the Legendre polynomials)

$$
\Psi=\sum_{n=0}^{\infty} \frac{a_{n}}{r^{n+1}} P_{n}(\cos \theta) .
$$

Several works have been devoted [2, 7], 10, 9, to establishing a relationship between this set of Weyl coefficients and the Relativistic Multipole Moments (RMM) of the solution, in an attempt to provide a mechanism for selecting solutions with physical meaning from the Weyl family. Naturally, the Schwarzschild solution, which is spherically symmetric, belongs to that family and its set of Weyl coefficients (9) is known [4]. Alternatively, the Erez-Rosen-Quevedo (ERQ) representation [3], 21] of the static and axisymmetric vacuum solutions in prolate spheroidal coordinates has another set of coefficients $\left\{q_{n}\right\}$ (obviously related with $\left\{a_{n}\right\}$; see [4 for details), whose values associated with the Schwarzschild solution are $\left\{q_{0}=1, q_{n}=0 \forall n\right\}$. An evident advantage of this representation is a simpler form of the metric function that describes the spherically symmetric solution. In [5] the suitability of these coordinates was discussed for describing spherical symmetry in General Relativity (GR) because the coordinates are harmonic for that class of metrics.

The identification of a given solution among all the solutions from either the Weyl family or the ERQ representation is a procedure that is neither unique nor easy to develop, despite the work done. That is due to the difficulties involved in establishing a relationship between the RMM of the solution and the Weyl coefficients, specially for solutions with a relevant physical meaning. In addition, the interpretation of a solution, once constructed or chosen from the Weyl family, is not provided by a well-known and definitively established procedure, unless the solution is constructed with the desired RMM. In this case, the parameters of a particular solution acquire a physical meaning by means of the RMM. Nevertheless, we have to deal with a set of infinite coefficients and quite complicated expressions for the metric functions of the line element. 
The aim of the paper is dual: first, we develop a class of solutions of the Weyl family with a very interesting physical meaning -the so called Linearized Multipole (LM) Solutions- because they are constructed as the solutions that have a fixed and finite number $(g+1)$ of RMM. The assumption that all those multipoles are quantities of a very small magnitude is taken into account. With this approach, used for the calculation of the specific Weyl coefficients, we obtain exact solutions of the static and axisymmetric Einstein vacuum equations with the physical meaning explained above. The M-Q(1) solution [7] is recovered for the case $g=1$. We gain a new solution that is able to describe relativistic gravitational fields very close to the Schwarzschild solution and hence it might become a very useful tool for researching the eventual physical effects due to deviations from spherical symmetry [17], [18.

Second, we shall attempt to give an interpretation of these solutions by means of the gravitational potential of a Newtonian object. As is known, the Schwarzschild solution can be viewed as a bar of length $2 m$ with constant linear density since the gravitational potential of such an object equals the metric function of the Monopole solution. Although this representation of a spherically-symmetric GR solution is disconcerting, it at least provides us with a characterization of the Monopole solution by means of the gravitational field of a body that in Newtonian Gravity (NG) would be a spherical distribution of mass $m$. The role played by that spherical distribution in NG is adopted by a non-spherical object (the constant-density bar) to describe the space-time with spherical symmetry in GR. The questions addressed here are as follows: How could a solution of the Weyl family be identified with some gravitational potential? Is there any physical object available to depict the solution defined by means of the metric function $\Psi$ ? What characteristics of that object are appropriate for representing that solution and what can they be used for? If we identify the spherically symmetric solution with a bar of constant linear density, what could we identify the other solutions with?

In [6] Letelier gives a physical interpretation of the multipolar ERQ solutions of the Einstein vacuum equations in terms of bars, and the author finds that each "multi-pole" corresponds to the Newtonian potential of a bar with a linear density proportional to a Legendre polynomial. First of all, we must point out that what he calls "multi-pole" has nothing to do with a solution of the Einstein equations possessing a single RMM, except for the Monopole case. However, this work establishes a relationship between each 
coefficient $\left\{q_{n}\right\}$ of the ERQ representation and a bar with a certain linear density. Since each static and axisymmetric vacuum solution can be written in the ERQ representation, we can obtain an interpretation of the series that defines the solution as the infinite sum of potentials associated with bars of equal length and certain densities [6]. Nevertheless, we shall see that this definition has important limitations and it proves to be useless for constructing a linear density of a single bar that allows us to describe the whole series of the solution in general cases.

The question of looking for an interpretation of the metric function $\Psi$ is developed in the case of the LM solutions. The conclusion we obtain leads to the adoption of an object slightly different to a single bar that plays the role of being the Newtonian representation of these solutions: A dumbbell, i.e., an object consisting of a bar and two identical masses, one at each end of the bar, will identify the LM solutions. The gravitational potential of such an object provides the metric function of the LM solutions by conveniently changing the linear density of the bar as well as the magnitudes of the masses. It must be pointed out here that this work provides a method for calculating both the linear density and the masses of the dumbbell in terms of the RMM possessed by the LM solution. Therefore, the differences with respect to the Schwarzschild solution, which is recovered from the LM solution by taking all RMM higher than the monopole equal to zero, can be specified explicitly. A detailed study of the bounds on the magnitude of the RMM imposed by the condition of a well defined positive linear density is carried out.

Finally we wish to stress some reasons why we think that this representation is useful and physically interesting. The relevant metric function of the LM solution is calculated from the density of the dumbbell, which is an even polynomial of $g$ degree. Therefore, the complete solution is defined by $g$ independent coefficients alone. This is a very simple way of describing a family of static and axisymmetric vacuum solutions rather than the Weyl or the ERQ representations. With the dumbbell we are introducing a generalization of the Newtonian representation of the Schwarzschild solution. Furthermore, we can use the dumbbell to describe some physical properties of the relativistic solution. We shall show that the positive-definite condition of the density allows us to decide whether the source that generates the space-time can be described as a flattened or elongated isolated body with respect to the spherical mass distribution. In addition, the existence of a connected horizon on the infinite-redshift surface $g_{00}=0$ can be described by means of a well-behaved density condition. 
The paper is organized as follows: in section 2 we explicitly calculate the expression for the Newtonian multipole moments of a bar of finite length with a linear density as well as the gravitational potential of such a mass distribution. In subsection 2.2 we first analyze the definition introduced by Letelier [6] and we claim the existence of a Newtonian representation of the Weyl solutions in analogy with the case of spherical symmetry.

In section 3 we present the LM solution. Its metric function and its Weyl coefficients are shown, and we introduce the dumbbell as a suitable object to represent this solution. We explicitly calculate its characteristics (density and masses) in terms of the RMM. The section is completed with a detailed analysis of the properties of the dumbbell and the calculation of its gravitational potential. Section 4 is devoted to showing some examples of special physical interest: we make a comparison between their linear densities and we look for bounds on the values of the RMM. The Erez-Rosen solution, the $\mathrm{M}-\mathrm{Q}^{(1)}$ solution and a particular case of the LM solution are studied. A Conclusions section reports an analysis of the results obtained and discussions about their relevance, together with future extensions of the work. We also include an Appendix that is used to show that the definition of density introduced by Letelier can be recovered by an alternative procedure.

\section{Description and interpretation of relativis- tic solutions by means of a Newtonian grav- itational potential and its multipole mo- ments}

\subsection{Newtonian gravity}

Let us consider the Newtonian gravitational potential of a mass distribution with density $\rho(\overrightarrow{\hat{z}})$, given by the following solution of the Poisson equation (we use units in which the gravitational constant $G=1$ )

$$
\Phi(\vec{x})=-\int_{V} \frac{1}{R} \mu(\overrightarrow{\hat{z}}) d^{3} \overrightarrow{\hat{z}}
$$

where the integral is extended over the volume of the source, $\overrightarrow{\hat{z}}$ is the vector that gives the position of a generic point inside the source, and $R$ is the 
distance between that point and any exterior point $P$, which is defined by its position vector $\vec{x}$. Let us now make an expansion of this potential in a power series of the inverse of the distance from the origin to the point $\vec{P}(r \equiv|\vec{x}|)$ by means of a Taylor expansion of the term $\frac{1}{R}$ around the origin of coordinates, where $R \equiv \sqrt{\left(x^{i}-\hat{z}^{i}\right)\left(x_{i}-\hat{z}_{i}\right)}$. For the case of an axially symmetric mass distribution, this multipole development leads to a Newtonian potential with the same form as equation (11) but with the Weyl coefficients $\left\{a_{n}\right\}$ replaced by $-M_{n}^{N G}$, which are parameters that denote the massive multipole moment of order $n$ that can be defined by means of an integral expression extended to the volume of the source,

$$
M_{n}^{N G}=2 \pi \iint \hat{z}^{n+2} \mu(\hat{r}, \hat{\theta}) P_{n}(\cos \hat{\theta}) \sin \hat{\theta} d \hat{\theta} d \hat{r}
$$

$\hat{r} \equiv|\overrightarrow{\hat{z}}|$ representing the radius of the integration point and $\hat{\theta}$ the corresponding polar angle.

From the next section onwards we shall make use of a source such as a bar of length $2 L$, centered and located along the $Z$ axis. There is a well-established framework in Newtonian Gravity (NG) for handling distributional line-sources such as this, and we shall therefore consider an object described by a line singularity on the $Z$ axis with the following linear density:

$$
\mu(\overrightarrow{\hat{z}})=\frac{1}{2 \pi} \frac{\delta(\hat{\rho})}{\hat{\rho}} \mu(\hat{z}),
$$

for some non-negative function $\mu(\hat{z}), \delta(\hat{\rho})$ being the Dirac function $\delta\left(\hat{\rho}-\hat{\rho}_{0}\right)$ at $\hat{\rho}_{0}=0$ and where $\{\overrightarrow{\hat{z}}\} \equiv\{\hat{\rho}, \hat{z}\}$ are cylindrical coordinates. Consequently, from equation (2) the gravitational potential of such a mass distribution is as follows:

$$
\Phi(\vec{x})=-\int_{V} \frac{1}{R} \mu(\overrightarrow{\hat{z}}) d^{3} \overrightarrow{\hat{z}}=-\int_{-L}^{L} \frac{\mu(\hat{z})}{\sqrt{\rho^{2}+(z-\hat{z})^{2}}} d \hat{z}
$$

where the position vector $\vec{x}$ is given by coordinates $(\rho, z)$, and $\overrightarrow{\hat{z}}$ is located along the $Z$ axis.

According to equation (3) the Newtonian multipole moments of this ob- 
ject (if the function $\mu(\hat{z})$ is even in $\hat{z}$ ) are as follows:

$$
M_{2 n}^{N G}=\int_{-L}^{L} \hat{z}^{2 n} \mu(\hat{z}) d \hat{z}=L^{2 n+1} \int_{-1}^{1} X^{2 n} \mu(L X) d X .
$$

\subsection{Interpretation of axisymmetric and static solutions of the Einstein vacuum equations}

As is known, the line element of a static and axisymmetric vacuum space-time is represented in Weyl form as follows

$$
d s^{2}=-e^{2 \Psi} d t^{2}+e^{-2 \Psi}\left[e^{2 \gamma}\left(d \rho^{2}+d z^{2}\right)+\rho^{2} d \varphi^{2}\right]
$$

where $\Psi$ and $\gamma$ are functions of the cylindrical coordinates $\rho$ and $z$ alone. The metric function $\Psi$ is a solution of the Laplace equation $(\triangle \Psi=0)$, and the other metric function $\gamma$ satisfies a system of differential equations whose integrability condition is merely the equation for the function $\Psi$. The Weyl family of solutions with good asymptotic behaviour are given in spherical coordinates $\{r, \theta\}$ as the series (1).

From the point of view of understanding the relativistic properties of the line element corresponding to any particular choice of the potential function $\Psi$, what is important, of course, is not the Newtonian moments $a_{n}$ but the RMM of the solution, first defined for static and axisymmetric vacuum solutions by Geroch [15] and Thorne [16]. The Newtonian moments were first expressed as functions of the RMM by Fodor, Hoenselaers and Perjés [14, and by Bäckdahl and Herberthson later [2], [11]. Although the full FHP relations are extremely complicated, they can be used to obtain relatively simple formulas for the coefficients $\left\{a_{n}\right\}$ in situations where the deviation of the relativistic solution from spherical symmetry is small. Some authors have devoted their work to this research [4], [7], [11]. In this sense, the spherically symmetric line element (i.e. the Schwarzschild solution) only has one RMM, the Monopole, and its metric function $\Psi$ is represented in Weyl coordinates by a bar of constant linear density of length $2 M, M$ being the mass of the Monopole solution. This interpretation is derived from the fact that the metric function $\Psi$ of the solution is equal to the Newtonian gravitational

\footnotetext{
${ }^{1}$ An identical conclusion can be derived if we approximate the source by a series of cylinders of radius $\epsilon$ and then take the limit $\epsilon \rightarrow 0$ or, equivalently, we consider the factor $\epsilon / L$ negligible since we are dealing with a very narrow bar $(\epsilon<<2 L)$.
} 
potential of such an object, i.e, if we take $\mu(\hat{z})=1 / 2$ in (5) we obtain the metric function of the Schwarzschild solution in Weyl coordinates [4]

$$
\Psi=\Phi=\frac{1}{2} \ln \left(\frac{z+M-\sqrt{\rho^{2}+(z+M)^{2}}}{z-M-\sqrt{\rho^{2}+(z-M)^{2}}}\right) .
$$

Moreover, it is also known [4], [9] that the set of infinite coefficients $\left\{a_{n}\right\}$ corresponding to the Schwarzschild solution are 2

$$
a_{2 n}=-M^{2 n+1} /(2 n+1), a_{2 n+1}=0 .
$$

These quantities are precisely equal (up to a sign) to the Newtonian multipole moments $M_{2 n}^{N G}$ of the bar with linear density $\mu=1 / 2$, and they can be explicitly calculated 3 from expression (6). Therefore, the identification between the metric function of the relativistic solution and the gravitational potential of the Newtonian mass distribution leads to a curious interpretation of the spherically symmetric solution of the Einstein vacuum equations. Let us remember that if $\Psi$ is generated by a Newtonian point particle with mass $M$ lying at the origin of coordinates so that it has the spherical form $\Psi=-M / \sqrt{\rho^{2}+z^{2}}$, then the corresponding line element describes the Curzon metric [12], which is not spherically symmetric.

In spite of the rather strange nature of this representation of the Monopole Solution in GR, very distant from Newtonian common sense, this identification with a Newtonian object may be useful to characterize solutions of the Weyl family, and at the same time it reveals how different the multipole moments are from NG to GR. Hence, we can assume that a physical object whose Newtonian moments equal the coefficients $\left\{a_{n}\right\}$ of the Weyl solution allows us to identify this solution since the Newtonian gravitational potential $\Phi$ of the object resembles the metric function $\Psi$ of the relativistic solution. The question arising here is whether we could arrange a similar identification for any other Weyl solution in analogy with the spherical symmetry case.

In [6] the author shows that the series

$$
\Psi=-\sum_{k=0}^{\infty} q_{k} Q_{k}(x) P_{k}(y)
$$

\footnotetext{
${ }^{2}$ Note that the expression for these coefficients can easily be verified by developing the metric function (8) in power series of $1 / r$ and identifying the expression with (2).

${ }^{3}$ Let us note that we have to take the half-length of the bar $L$ equal to $M$.
} 
can be interpreted as the infinite sum of potentials associated with bars of equal length $2 \sigma$ and linear densities $\lambda_{k}(z)=\frac{1}{2} q_{k} P_{k}(z / \sigma)$. The expression (10) is the metric function, written in prolate spheroidal coordinates $\{x, y\}$ (see equation (20.6) in page 305 of [20] for the definition of these coordinates) corresponding to the ERQ representation [3] of the axisymmetric static vacuum equations, where $P_{k}(y)$ and $Q_{k}(x)$ stand for the Legendre polynomials and associated Legendre functions of the second kind respectively. It is clear, from the linearity of the Laplace equation, that this conclusion concerning the infinite sum of axial bars generating the potential (10) is equivalent to the statement that it can also be generated by a single bar with linear density:

$$
\lambda(z)=\frac{1}{2} \sum_{k=0}^{\infty} q_{k} P_{k}(z / \sigma),
$$

where $q_{k}$ are the coefficients of the ERQ representation corresponding to a particular solution (10). In fact, the result obtained by Letelier can be recovered by means of considerations other than those used in [6]. We only need to use the relation between the sets of coefficients of both the Weyl and the ERQ representations [9], 4], concluding that equations (5) and (6) mean that the Newtonian multipole moments of the bar with its density given by $\lambda(z)$ (11) are precisely the coefficients $a_{2 n}$ of the Weyl family of solutions $\left(M_{n}^{N G}=-a_{n}\right)$, and the Newtonian gravitational potential is given by the Weyl series (11). The detailed calculations are addressed in the Appendix.

Nevertheless, this linear density (11) is useless in the general case and, for several reasons, inefficient for describing the mass distribution of the desired Newtonian objects. First, we point out that except for the cases where the set of ERQ coefficients $\left\{q_{k}\right\}$ of the solution is finite, the expression for that linear density cannot be summed explicitly. The cases of the Schwarzschild solution, defined by $q_{0}=1, q_{k}=0, \forall k>1$, as well as the Erez-Rosen solution [8], which only has two coefficients $q_{k}$ different from zero $\left(q_{0}=1, q_{2} \neq 0\right.$, $q_{k}=0, \forall k>2$ ) are, of course, good examples of this. Second, the definition of the density (11) associated with a wide class of solutions of the Weyl family (all the reflection-symmetric solutions for instance) shows a specific anomaly at both ends of the bar $(t= \pm 1, z= \pm \sigma= \pm M)$ where the value of the density diverges unless the series of the coefficients $\left\{q_{n}\right\}$ itself converges:

$$
\lambda(z= \pm \sigma)=\frac{1}{2} \sum_{k=0}^{\infty} q_{2 k} .
$$


Since the aim of the work is to extend the construction of an object with a representative density to a wide range of solutions, the establishment of a relation between the multipole structure of the Weyl solution and the density of the object would be a very relevant success. This achievement would allow us to describe how the corrections to the spherical symmetry of the Weyl solution are reflected in a non-constant density of the mass distribution. In our opinion, the efforts of such research should be focused on identifying the so-called Pure Multipole Solutions [7], 9], [10] because these solutions attempt to describe deviations from spherical symmetry by means of their Multipole Moments.

In the next section we introduce a new family of static and axisymmetric solutions of the Einstein vacuum field equations with a specifically known multipolar structure, and we derive for it an interpretation of its metric function $\Psi$ by means of the Newtonian gravitational potential of a particular mass distribution. This physical object with its characteristics (like its density) is a sort of mathematical artifice 4 used to describe the relativistic solution and it should not be confused with the real non-spherical isolated source that generates the exterior gravitational field.

\section{Solutions of the Weyl family with a pre- scribed relativistic multipole structure}

\subsection{The solution}

In [9], the solution of the Weyl family that represents the exterior gravitational field of a mass distribution whose multipole structure only possesses mass $M$ and quadrupole moment $Q$ was shown. The procedure for constructing this M-Q solution requires knowledge of the relativistic multipole moments (RMM) of a generic Weyl solution; that is to say, we need to calculate the relationship between the RMM and the coefficients $\left\{a_{n}\right\}$ of the Weyl family of solutions. This calculation is done by means of the FHPmethod [14] and the coefficients $\left\{a_{n}\right\}$ obtained can be constrained to satisfy the conditions imposed on the RMM claimed for the solution. The M-Q solution has become a useful tool for describing small deviations from the

\footnotetext{
${ }^{4}$ Let us note that expression (5), obtained from equation (44), is identical to that derived from the method of singular sources (see 22] and references therein) used to construct relativistic static solutions with a singular source.
} 
spherically symmetric solution [17], [18]. The assumption considered for the M-Q solution is that $Q$ is small since we want to approach the Schwarzschild solution as closely as possible, and all the RMM of higher order are negligible 5 . Thus, the M-Q solution is constructed as a sum of functions in a power series of the dimensionless quadrupole parameter $q \equiv Q / M^{3}$ starting at the Schwarzschild solution as the first order, in such a way that the successive powers of $q$ control the desired corrections to the spherical symmetry.

We now wish to introduce a similar but different proposal for constructing relativistic gravitational fields close to the Schwarzschild solution. When attempting to describe an isolated compact body that is not spherically symmetric, all the RMM appear no matter how small the deviation is from being spherical. Therefore, let us assume that all RMM appear in the solution that we want to construct but let us restrict their magnitudes to being very small, in such a way that we can neglect all terms in the Weyl coefficients wherever a product of RMM is involved. This explains the name of the family of solutions: Linearized Multipole (LM) solution. The structure of the Weyl coefficients for that solution is as follows:

$$
a_{2 n}^{L M}=-M^{2 n+1} \sum_{k=0}^{g} m_{2 k} F_{k}(n), a_{2 n+1}=0
$$

where (equatorial symmetry is assumed) $m_{2 k} \equiv \frac{M_{2 k}}{M^{2 k+1}}$ denotes the dimensionless parameter associated with the multipole moment $M_{2 k}$ of order $2 k, g$ being the number of RMM in the solution in addition to the monopole, and

\footnotetext{
${ }^{5}$ This consideration about the RMM higher than $Q$ is supported by the following argument: The Newtonian calculation of the multipole moments of an ellipsoidal mass distribution leads to the conclusion that as they increase their order they decrease in magnitude proportionally to the powers of the eccentricity of the ellipsoidal configuration (see 4 for details).

${ }^{6}$ The case of the solution M-Q ${ }^{(1)}$ 7] can be recovered with the first two terms $(g=1)$. The function $F_{1}(n)$ reduces to the corresponding expression in 7]. The explicit expression (14) is deduced from the calculation of the RMM of a generic static and axisymmetric solution by applying the FHP method.
} 
the functions $F_{k}(n)$ for each $2^{2 k}$-pole moment are defined by the expression 7 :

$$
F_{k}(n)=\left\{\begin{array}{l}
I(k) \frac{n !(2 n-1) ! !}{(n-k) !(2 n+2 k+1) ! !}[k n+I I(k)], \quad 1<k \leq n \\
\frac{1}{2 n+1}, \quad k=0
\end{array}\right.
$$

with

$$
\begin{aligned}
I(k) & =\frac{1}{2^{k-1} k(k+1)}\left(\begin{array}{c}
4 k+1 \\
2 k
\end{array}\right) \\
I I(k) & =\frac{k}{2}\left(2 k^{2}+k+1\right) .
\end{aligned}
$$

We decompose expression (14) in the following form:

$$
F_{k}(n)=h(k)+\sum_{j=0}^{k} \frac{h_{j}(k)}{2 n+2 j+1},
$$

where $h(k)$ and $h_{j}(k)$ are coefficients depending on the $2^{2 k}$-pole moment. The independent term of this decomposition, $h(k)$, is easily determined by the following expression, since the numerator of each $F_{k}(n)$ is a polynomial of degree $k+1$ :

$$
h(k)=k \frac{I(k)}{2^{k+1}}, \forall k>0,
$$

whereas the other coefficients $h_{j}(k)$ must satisfy the following relations:

$$
h_{j}(k) \prod_{i=0, i \neq j}^{k}\left(2 n_{j}+2 i+1\right)=I(k)\left(k n_{j}+I I(k)\right) \prod_{i=0}^{k-1}\left(n_{j}-i\right), j=0 \ldots k
$$

where $n_{j} \equiv-\frac{2 j+1}{2}$. An easy, but cumbersome, calculation of the products appearing in (18) leads to the following explicit expression for the coefficients $h_{j}(k)\left(\forall k \geq j\right.$, since $h_{j}(k)=0$ for $\left.k<j\right)$ :

$$
h_{j}(k)=\frac{1}{2^{4 k-1}}(-1)^{k-j-2}\left(\begin{array}{c}
4 k+1 \\
2 k
\end{array}\right) \frac{k^{2}+k / 2-j}{(k+1)} \frac{(2 k+2 j) !}{(2 j) !(k+j) !(k-j) !} .
$$

With respect to the case of the function $F_{0}(n)$ we obviously have $h(0)=0$ and $h_{0}(0)=1$.

\footnotetext{
${ }^{7}$ Note that $F_{k}(n)=0$ for $k>n$.
} 


\subsection{Characterization of the solution}

\section{A) The density of the bar.}

In analogy with the homogeneous axial rod of the Schwarzschild solution, we wish to introduce a non-constant density on an axial rod whose Newtonian gravitational potential reproduces the metric function of the LM solution, and whose Newtonian multipole moments lead to the Weyl coefficients of the solution. These coefficients (13) $\left\{a_{n}\right\}$ of the LM solution are given by the following expression

$$
a_{2 n}^{L M}=-\sum_{j=0}^{g} \frac{M^{2 n+2 j+1}}{2 n+2 j+1} \frac{H_{j}}{M^{2 j}}-M^{2 n+1} H,
$$

where we have made use of the decomposition (16) of $F_{k}(n)$, and the following definitions:

$$
H \equiv \sum_{k=0}^{g} m_{2 k} h(k) \quad, \quad H_{j} \equiv \sum_{k=j}^{g} m_{2 k} h_{j}(k) .
$$

It is easy to see that if we take $\mu(\hat{z})=c_{j} \hat{z}^{2 j}$ for an arbitrary constant $c_{j}$, then the corresponding Newtonian moments from equation ([6), with the notation $\hat{z} \equiv X L$, come out as

$$
M_{2 n}^{N G}=L^{2 n+1} \int_{-1}^{1} X^{2 n} \mu(L X) d X=\frac{2}{2 n+2 j+1} L^{2 n+2 j+1} c_{j} .
$$

and the choice $\mu(\hat{z})=c L \delta(\hat{z} \pm L)$ leads to

$$
M_{2 n}^{N G}=L^{2 n+1} c \text {. }
$$

Therefore, the condition $a_{2 n}=-M_{2 n}^{N G}$ for the coefficients (201) is automatically satisfied if the physical object that represents the LM solution is, let us say, a dumbbell consisting of two point-like masses of magnitude $\frac{H M}{2}$ located at both ends of a bar 8 of half-length $L=M$, whose linear density is constructed with the coefficients $H_{j}$ (21) as follows:

$$
\mu(X)=\frac{1}{2} \sum_{j=0}^{g} X^{2 j} H_{j} \quad, X \in[-1,1] .
$$

\footnotetext{
${ }^{8}$ These masses arise from a function $\mu(X)=\frac{1}{2} H[\delta(X+1)+\delta(X-1)]$ (see equation (23)) considered in addition to the linear density of the bar of the dumbbell. An alternative argument comes from the direct comparison of the gravita-
} 
Before continuing to analyze the properties of the linear density of the bar $\mu(X)$, the following must be emphasized again: We claim to represent a certain type of Weyl solutions by means of the Newtonian gravitational potential of a physical object that resembles a dumbbell, whose characteristics are two identical masses and the non-constant density of the bar connecting both of them. It is true that this paradigm is only a mathematical convenience, and the dumbbell must not be regarded as representing a real physical mass distribution lying along the $Z$ axis of the space-time. Nevertheless, the implementation of this representation is, as we shall see in the following sections, a useful tool for describing the physical characteristics of a static and axisymmetric space-time.

First, with this picture in mind we generalize the known feature of the Schwarzschild solution to the LM solution that represents a relativistic Weyl solution whose first $g$ RMM, in addition to the monopole moment, are nonzero. The difference with respect to the Schwarzschild solution consists of the change in the density of the bar and the addition of masses. Within this representation no divergence such as that appearing in the definition of the density made by Letelier [6] occurs, and the dumbbell admits a well-behaved linear density for its bar.

Second, the following are very important points of this result: on the one hand, the density (24) completely defines the whole structure of the dumbbell since the additional masses can be calculated from it, as we shall see in the next section. On the other hand, this density is constructed in terms of the RMM of the source and hence it provides a mechanism for adjusting the contributions of different RMM to the changing aspect of the physical object that represents the non-spherical behaviour of the relativistic solution.

In the following section we shall investigate the properties of the density (24) in detail and the interpretation of the masses of the dumbbell. In section 4 we implement some examples and provide relevant conclusions from

tional potential of two particles of mass $m$ situated at distances $-L$ and $L$ respectively along the $Z$ axis, $\Psi=\Phi=-\frac{m}{\sqrt{\rho^{2}+(z+L)^{2}}}-\frac{m}{\sqrt{\rho^{2}+(z-L)^{2}}}$, and the corresponding metric function by means of performing a power series expansion as follows: $\Phi=-\frac{m}{\sqrt{\rho^{2}+(z+L)^{2}}}-\frac{m}{\sqrt{\rho^{2}+(z-L)^{2}}}=-\sum_{n=0}^{\infty} \frac{P_{2 n}(\omega)}{r^{2 n+1}}\left(2 m L^{2 n}\right)$, and therefore the Weyl coefficients related to this potential of two particles are $a_{2 n}=-2 m L^{2 n}$. Let us note that $\frac{1}{\sqrt{\rho^{2}+(z-x)^{2}}}=\frac{1}{r} \sum_{n=0}^{\infty}\left(\frac{x}{r}\right)^{n} P_{n}(z / r)$. 
comparisons among the different cases.

\section{B) Properties and characteristics of the dumbbell.}

i) The density of the bar at its ends equals the value of the density for the spherical case (Schwarzschild), i.e., $\mu(X= \pm 1)=1 / 2$ since the following relation is fulfilled:

$$
\sum_{j=0}^{g} H_{j}=1
$$

It is easy to see that (25) holds because the structure of the functions $F_{k}(n)$ is such that the sum of all the coefficients $h_{j}(k)$ for each $k$ (multipole order) vanishes:

$$
\sum_{j=0}^{g} h_{j}(k)=0 \quad, k=0 \ldots g .
$$

ii) The masses of the dumbbell have been calculated previously as $M H / 2$. Since we explicitly know the value of $h(k)$ for each multipole moment (17), we can rewrite and evaluate the mass of each ball as follows:

$$
H=\sum_{k=0}^{g} \frac{m_{2 k}}{2^{2 k}(k+1)}\left(\begin{array}{c}
4 k+1 \\
2 k
\end{array}\right) \text {. }
$$

Alternatively, we can calculate the mass of the balls by means of the excess or deficit (depending on the sign of the RMM) provided by the density of the bar with respect to the total mass $M$ of the solution. The Newtonian zero-order multipole moment of the bar is given by the following expression $(L \equiv M)$ :

$$
a_{0}^{b a r}=-\int_{-L}^{L} \mu(z) d z=-2 L \int_{0}^{1} \mu(X) d X=-M \sum_{j=0}^{g} \frac{H_{j}}{2 j+1},
$$

and it represents the mass of the bar except for the sign. Therefore, the following relation must hold for the total mass of the dumbbell:

$$
-a_{0}^{b a r}+2 \nu=M,
$$

where $\nu$ denotes the mass of each ball of the dumbbell, and hence we have

$$
\nu=\frac{M}{2}\left[1-\sum_{j=0}^{g} \frac{H_{j}}{2 j+1}\right] \text {. }
$$


In fact, this expression is true, i.e., we can prove that $\nu=M H / 2$ from the structure of the functions $F_{k}(n)$ : the equivalence between $\nu$ (30) and $M H / 2$ (27) leads to

$$
1-\sum_{j=0}^{g} \frac{1}{2 j+1} \sum_{k=j}^{g} h_{j}(k) m_{2 k}=\sum_{k=0}^{g} h(k) m_{2 k},
$$

or equivalently, for each multipole order $k>0$ we have that $\left(h_{0}(0)=1\right.$ and $h(0)=0$ since $\left.m_{0}=1\right)$

$$
\sum_{j=0}^{g} \frac{h_{j}(k)}{2 j+1}=-h(k) \quad, \quad \forall k=1 \ldots g
$$

a relation that is fulfilled for every $k$ since $F_{k}(n=0)=0$.

In conclusion, the dumbbell representing the LM solution is characterized exclusively by the linear density of its bar (24). The mass of each ball of the dumbbell is given by $\nu$ (30). Accordingly, the set of coefficients $\left\{H_{j}\right\}$ determines the Weyl solution and characterizes it for the $2^{2 g}$-pole order considered. We work with $g+1$ coefficients but owing to the constraint, (25), we only have $g$ independent coefficients $H_{j}$, and so we construct the LM solution having $g$ multipole moments in addition to the monopole with $g$ independent coefficients in accordance with the $g$ dimensionless multipole moments $m_{2 k}$.

\section{C) The metric function as a gravitational potential.}

The interpretation of the LM solution by means of the gravitational potential of the dumbbell allows us to calculate the metric function of the solution as follows

$$
\Psi=\Phi=\int_{-1}^{1} \frac{-M \mu(X)}{\sqrt{\rho^{2}+(z-M X)^{2}}} d X-\frac{\nu}{\sqrt{\rho^{2}+(z-M)^{2}}}-\frac{\nu}{\sqrt{\rho^{2}+(z+M)^{2}}}
$$

with $\mu(X)$ the linear density (24) of the bar of the dumbbell and $\nu$ being the mass of each ball (30). Since the density $\mu(X)$ is a polynomial we have to evaluate the integrals of the following type:

$$
\Upsilon_{\alpha} \equiv \int_{-1}^{1} \frac{X^{\alpha}}{\sqrt{\rho^{2}+(z-M X)^{2}}} d X
$$

using the recurrence relation

$$
\Upsilon_{\alpha}=\left.\Gamma_{\alpha}(X) \sqrt{\rho^{2}+(z-M X)^{2}}\right|_{-1} ^{1}+\Lambda_{\alpha} \Upsilon_{0} \quad, \quad \alpha \geq 1,
$$


where $\Gamma_{\alpha}(X) \equiv \sum_{i=0}^{\alpha-1} p_{i}(\alpha) X^{i}$ is a polynomial of degree $\alpha-1$ and $\Lambda_{\alpha}$ a function of the Weyl coordinates $\{\rho, z\}$ not depending on the variable $X$. A cumbersome calculation leads to the following iterative scheme $\left(r \equiv \sqrt{\rho^{2}+z^{2}}\right.$ denotes the radial Weyl coordinate):

$$
\begin{aligned}
& \Lambda_{\alpha}=M z p_{0}(\alpha)-r^{2} p_{1}(\alpha) \\
& p_{\alpha-1}(\alpha)=\frac{1}{\alpha M^{2}} \quad, \quad p_{\alpha-2}(\alpha)=\frac{z(2 \alpha-1)}{\alpha(\alpha-1) M^{3}} \\
& 0=r^{2}(\alpha-k) p_{\alpha-k}(\alpha)-z M(2 \alpha-2 k-1) p_{\alpha-(k+1)}(\alpha)+ \\
& +M^{2}(\alpha-k-1) p_{\alpha-(k+2)}(\alpha) \quad, \forall k \geq 1, \alpha \geq 1,
\end{aligned}
$$

and the integral

$$
\Upsilon_{0}=\frac{1}{M} \ln \left(\frac{z-M-r_{-}}{z+M-r_{+}}\right) \quad, \quad r_{ \pm} \equiv \sqrt{\rho^{2}+(z \pm M)^{2}}
$$

Finally, the metric function is given by the following expression, where we obviously recover the Schwarzschild solution for the case $g=0$ :

$$
\begin{aligned}
\Psi=\Phi & =-\frac{M}{2} \sum_{j=0}^{g} \int_{-1}^{1} \frac{X^{2 j} H_{j}}{\sqrt{\rho^{2}+(z-M X)^{2}}} d X+ \\
& -\frac{\nu}{\sqrt{\rho^{2}+(z-M)^{2}}}-\frac{\nu}{\sqrt{\rho^{2}+(z+M)^{2}}}= \\
& =\frac{1}{2}\left[H_{0}+\sum_{j=1}^{g} H_{j} \Lambda_{2 j}\right] \ln \left(\frac{z+M-r_{+}}{z-M-r_{-}}\right)+ \\
& +\frac{M}{2}\left[\sum_{j=1}^{g} H_{j} \Gamma_{2 j}(-1)\right] r_{+}-\frac{M}{2}\left[\sum_{j=1}^{g} H_{j} \Gamma_{2 j}(1)\right] r_{-}-\nu\left[\frac{1}{r_{+}}+\frac{1}{r_{-}}\right]
\end{aligned}
$$

\section{Some examples and constraints to the mul- tipole moments}

\subsection{The Erez-Rosen $v s$ the $\mathrm{M}-\mathrm{Q}^{(1)}$ solution}

These are both two-parameter solutions of the Weyl family, one of the parameters $(M)$ representing the mass and the other $q$ or $q_{2}$ denoting the dimensionless quadrupole moment for the $\mathrm{M}-\mathrm{Q}^{(1)}$ or the ER solution respectively. 
In [17] a comparison between these solutions was developed and different conclusions were obtained regarding the behaviour of a gyroscope precessing in circular orbits in each space-time. The Weyl coefficients of the ER solution are known [4]:

$$
a_{2 n}^{E R}=-\frac{M^{2 n+1}}{2 n+1}\left(1+q_{2} \frac{2 n}{2 n+3}\right)=-M^{2 n+1}\left[\frac{1-q_{2} / 2}{2 n+1}+\frac{3 q_{2} / 2}{2 n+3}\right] .
$$

From this expression for the Weyl coefficients it immediately follows that the ER solution can be represented by the Newtonian gravitational potential of a bar with linear density:

$$
\mu^{E R}(X)=\frac{1}{2}\left(1-\frac{q_{2}}{2}\right)+\frac{3}{4} q_{2} X^{2},
$$

since the multipole Newtonian moments (6) of a bar with this linear density reproduce equation (39). As can be seen, this result recovers (11) Letelier's definition [6] for this solution because the ER solution has a finite number of coefficients $\left\{q_{n}\right\}$ in the ER representation. From the density $\mu^{E R}(X)$ we can also obtain the Newtonian potential (5),

$$
\begin{aligned}
\Phi=\Psi & =-\frac{1}{2}\left[1-\frac{q_{2}}{2}+\frac{3 q_{2}}{4 M^{2}}\left(2 z^{2}-\rho^{2}\right)\right] \ln \left(\frac{M-z+r_{-}}{-M-z+r_{+}}\right)+ \\
& -\frac{3 q_{2}}{8 M^{2}}\left[(3 z+M) r_{-}-(3 z-M) r_{+}\right]
\end{aligned}
$$

A straightforward calculation allows us to prove that this expression (41) reproduces the known metric function of the ER solution in prolate spheroidal coordinates (see equation (20.9) on page 306 of [20] for details.)

In contrast to the ER solution, the $\mathrm{M}-\mathrm{Q}^{(1)}$ solution entails a different representation. This solution is the linear order in the quadrupole parameter of the solution M-Q [9]. The Weyl coefficients of this solution are known [9]-[11]:

$$
a_{2 n}=-\frac{M^{2 n+1}}{2 n+1}\left(1+q \frac{5 n(n+2)}{2 n+3}\right)
$$

where the parameter $q \equiv \frac{Q}{M^{3}}$ is used to denote the dimensionless quadrupole moment. As we have previously noted, the sum of the series for the Letelier density (11) is not available is this case, since the set of ERQ coefficients 
$\left\{q_{n}\right\}$ of this solution is not finite (see [7] for details): $q_{0}=1, q_{2}=\frac{15}{2} q, q_{2 n}=$ $\frac{5}{4}(4 n+1) q, \forall n \geq 2$. From equation (6) and the Weyl coefficients (42) it is easy to see that the $\mathrm{M}-\mathrm{Q}^{(1)}$ solution can be represented by a dumbbell consisting of a bar of length $2 L$ with linear density 9

$$
\mu^{M-Q^{(1)}}(X)=\frac{1}{2}\left(1-\frac{15}{8} q\right)+\frac{15}{16} q X^{2}
$$

and a point-like particle at each end of the dumbbell with respective masses $\nu=\frac{5}{8} q M$.

We now compare both solutions, analyzing the corresponding linear densities of their bars, which are polynomials of the form $\mu(X)=A+B X^{2}$. The maximum or the minimum (depending on the sign of the quadrupole parameter, $q, q_{2}<0$ or $q, q_{2}>0$ respectively) of the density is located at the origin and is given by $\mu(0)=A$, as can be seen in Figure 1 .

The following remarks can be made about the behaviour of the density. The ER solution is represented by a bar with linear density (40) rather than a dumbbell, as is the case for the $\mathrm{M}-\mathrm{Q}^{(1)}$ solution. Let us note that we can evaluate the total mass of the solution by calculating the Weyl coefficient $a_{0}$ as the integral of the density along the bar, and we obviously obtain $a_{0}=-M$.

The density of the $\mathrm{M}-\mathrm{Q}^{(1)}$ solution is equal to $1 / 2$ at both ends of the bar, as shown in Figure 1. Therefore, for $q<0$ the linear density is positive definite along the whole bar and it has the maximum value at $z=0: \mu(X=$ $0)=A=\frac{1}{2}\left(1-\frac{15}{8} q\right)$, whereas for $q>0$ a suitable definition of the density requires the following constraint:

$$
A \geq 0 \Rightarrow q \leq \frac{8}{15} .
$$

The ER solution shows slight differences with respect to such behaviour: for $q_{2}<0$ we require $\mu(X= \pm 1) \geq 0$, which leads 10 to the following constraint on the quadrupole parameter:

$$
\frac{-1+q_{2} / 2}{3 / 2 q_{2}} \geq 1 \Leftrightarrow\left|q_{2}\right| \leq 1
$$

\footnotetext{
${ }^{9}$ The Weyl coefficients can be decomposed as follows: $a_{2 n}=-\frac{M^{2 n+1}}{2 n+1}\left(1-\frac{15 q}{8}\right)-\frac{M^{2 n+3}}{2 n+3}\left(\frac{15 q}{8 M^{2}}\right)-M^{2 n+1} \frac{5}{4} q$.

${ }^{10} \mathrm{Or}$, equivalently, we demand the roots of the polynomial to be greater than 1 (in absolute value): $|\sqrt{-A / B}| \geq 1$.
} 


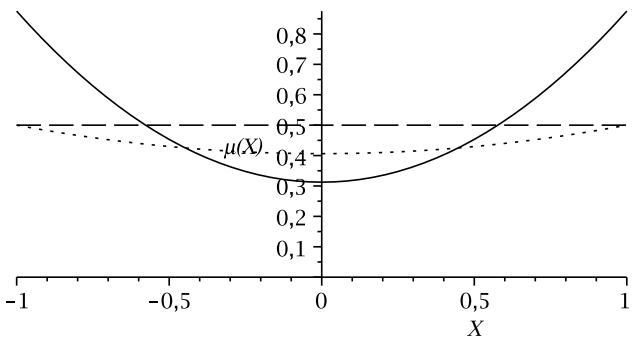

$\frac{- \text { Schwarzschild }- \text { Erez-Rosen } \cdots \cdots \mathrm{MQ}^{(1)}}{q=0.1}$

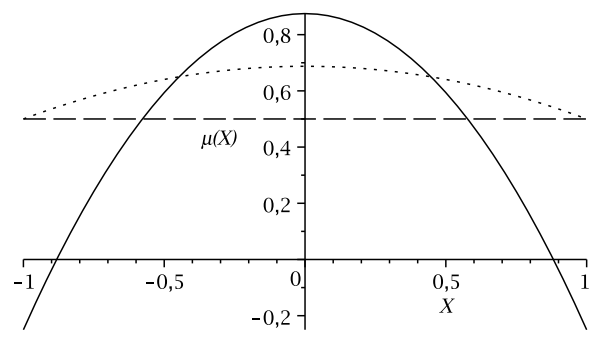

- - Schwarzschild $\cdots \cdots \mathrm{MQ}^{(1)}-$ Erez-Rosen

Figure 1: Linear density profiles for different values of the parameter $q$ corresponding to the $M-Q^{(1)}$ and Erez-Rosen solution compared with the Schwarzschild constant linear density. The positive sign of $q$ provides a density representing an elongated object whereas $q<0$ represents a flattened object, which agrees with a decrease or increase in the density at the center of the bar respectively.

whereas for the case $q_{2}>0$ we need $A \geq 0 \Leftrightarrow q_{2} \leq 2$.

In conclusion, the linear density of the $\mathrm{M}-\mathrm{Q}^{(1)}$ solution is positive definite for the range $q \in\left(-\infty, \frac{8}{15}\right]$ whereas the ER solution is characterized by a linear density that is positive definite for the range of the quadrupole parameter $q_{2} \in[-1,2]$. Since the relation between $q_{2}$ and $q$ is known, because they both represent the dimensionless quadrupole moment $\left(q_{2}=\frac{15}{2} q\right)$, the quadrupole moment $Q$ is constrained as follows:

$$
Q \in\left\{\begin{array}{rc}
M^{3}\left(-\infty, \frac{8}{15}\right], & M-Q^{(1)} \\
M^{3}\left[-\frac{2}{15}, \frac{4}{15}\right], & E R
\end{array}\right.
$$

The upper limit of $Q$ for the $\mathrm{M}-\mathrm{Q}^{(1)}$ solution is exactly twice the magnitude of that of the ER solution and it does not have a lower bound, whereas the quadrupole moment must be higher than a minimum value for the ER solution. 
As we already noted in the previous section, this representation of the Weyl solutions by means of the artificial construction of a dumbbell allows us to characterize the properties of the Weyl solution. Let us go deeper into this assessment by making an interpretation of the behaviour of the linear densities we have obtained.

i) In a vacuum relativistic solution, a negative value of the quadrupole parameter $q$ is associated with a flattened object whereas $q>0$ denotes an elongated source. With the representation of the Weyl solution by means of a dumbbell this characteristic of the source can be modeled by the behaviour of the bar density as follows: if $q>0$ the linear density of the bar shows a deficit of mass with respect to the constant linear density $\mu=1 / 2$ that corresponds to spherical symmetry (see Figure 1), at the same time as the masses of the point-like particles (the balls of the dumbbell) are positive. In the opposite case, for $q<0$ the linear density shows an excess of mass with respect to $\mu=1 / 2$, which is related to a negative value for the masses of the balls (deficit of mass at the ends of the bar). This behaviour of the density is therefore in concordance with the model picture of an elongated/flattened object in comparison with the spherically symmetric configuration.

ii) The ranges of values (46) obtained for $Q$ in both solutions are consistent since our family of LM solutions requires small values for their multipole parameters to maintain the physical interpretation we have provided for such solutions. However, these ranges should not be interpreted as bounds on the real values of the multipole moments that non-spherical bodies of anisotropic fluids, eventually considered as sources of these Weyl exterior solutions, could have. If the density is not positive definite this means that the method used to represent the potential $\Psi$ breaks down for values outside the ranges obtained.

iii) Nevertheless, some interpretation can be attempted regarding these ranges of values. It is known (see 21] for details) that the horizon of the Erez-Rosen metric, i.e., the hypersuface $x=1$ (where $x$ denotes the radial prolate coordinate, or equivalently $r=2 M$ in Schwarzschild coordinates) can be divided into two parts: the first part represents the horizon (where the norm of the time-like Killing vector is null) and the second corresponds to the Killing singularity, where that vector becomes infinite. For instance, if $0 \leq q_{2} \leq 2$ the horizon totally covers the hypersurface $x=1$, but for other values of the quadrupole parameter $q_{2}$ there is only a restricted range of values for the angular coordinate that makes $g_{00}^{E R}=0$ on the surface $x=1$. A detailed study 
of the infinite redshift surface $g_{00}=0$ was done in [4] (see web address to download file) with respect to the $\mathrm{M}-\mathrm{Q}^{(1)}$ solution. There it was established that there is a range of values for the quadrupole parameter $q$ where the horizon totally covers the hypersurface $x=1$. For values of $q$ outside this range that surface diverges for certain angular regions. The range of values of $q$ is exactly that obtained when we require the density of the dumbbell to be positive definite (46).

Thus, there is another feature of the dumbbell representation: a positive or negative value of the density allows us to characterize whether the surface $g_{00}=0$ completely covers the surface $x=1$ or not. If $\mu(\hat{z})>0$ (values of $q$ within the range), then $e^{2 \Psi} \equiv g_{00}=0$ for all values of the angular coordinate, whereas for $\mu(\hat{z})<0$ (values of $q$ outside the range) that surface diverges. The behaviour of the metric function $\Psi$ for different values of the density can be interpreted in that sense11: if $\rho$ tends to zero with $-M<z<M$, then $\Psi$ goes to $-\infty$ if the linear density $\mu(\hat{z})>0$ : i.e., when we are approaching the source $g_{00}$ tends to zero if the density is positive (the horizon is reached). By contrast, $\Psi$ tends to infinity if the density $\mu(\hat{z})<0$ when approaching the source, and hence $g_{00}$ tends to infinity, because we find the Killing singularity.

In conclusion, the positive-definite condition of the density is tantamount to guaranteeing that the vacuum solution possess a horizon that covers the hypersurface $x=1$ completely. Otherwise a naked singularity is found when approaching the source.

iv) A final comment on the dumbbell's representation. The Weyl series (11) can be rewritten as the following linear superposition of Curzon solutions:

$$
\Psi=\sum_{n=0}^{\infty} \frac{a_{n}}{r^{n+1}} P_{n}(\cos \theta)=\sum_{n=1}^{\infty} \frac{c_{n}}{\sqrt{\rho^{2}+\left(z-x_{n}\right)^{2}}},
$$

where the Curzon solution [12] corresponds to a point-like particle with mass $c_{n}$ located at the point $x_{n}$ on the $Z$ axis. Equation (47) for the metric function $\Psi$ requires the following relation between the Weyl coefficients and

\footnotetext{
${ }^{11}$ Note that the relation between the Weyl radial coordinate $R$ and the Schwarzschild coordinate $r$ or prolate radial coordinate $x$ is $R=\sqrt{r^{2}+M^{2} \cos ^{2} \theta-2 M r}=M \sqrt{x^{2}+y^{2}-1}$. Hence, the Schwarzschild surface $r=2 M(x=1)$ corresponds to the rod of the dumbbell since $R=M y$ (or equivalently $\rho=0, z=M y)$ and $-1 \leq y \leq 1$.
} 
the parameters $c_{n}, x_{n}$ :

$$
a_{n}=\sum_{x=1}^{\infty} x_{i}^{n} c_{i} .
$$

Well known results in Newtonian potential theory allow us to approximate a continuous line density with a series of point masses in such a way that the corresponding gravitational potential can be written as a linear combination of Curzon solutions. In fact, it is easy to see that a linear density of the type $\mu(z)=\sum_{i} c_{i} \delta\left(z-x_{i}\right)$ provides a potential $\Psi$ (477) in such a way that the relation (48) is satisfied from equation (6), and the continuous limit of the discrete density leads to the linear density constructed in the above cases for certain sets of the discrete parameters $\left\{x_{i}\right\},\left\{c_{i}\right\}$.

\subsection{The Monopole-Quadrupole- $2^{4}$-pole solution}

Analogously to the method previously used for other cases, the LM solution can be represented by the gravitational potential of a dumbbell with the linear density of its bar given by the following expression (see (24) and (21) for details):

$$
\mu^{L M}(X)=\frac{1}{2}\left[1-\frac{15}{8}\left(q-\frac{21}{4} m_{4}\right)+\frac{15}{8}\left(q-42 m_{4}\right) X^{2}+\frac{2205}{32} m_{4} X^{4}\right],
$$

and the masses of the particles at both ends of the bar are given by

$$
\nu=\frac{M}{8}\left(5 q+\frac{21}{2} m_{4}\right)
$$

This density shows a maximum or a minimum, depending on the sign of $H_{1}$, at the origin $\mu(X=0)=\frac{1}{2} H_{0}$ : if $H_{1}>0$, or equivalently if $q-42 m_{4}<0$, then the density possess a maximum and two minima at both symmetric points $X_{\text {min }}= \pm \sqrt{\frac{-H_{1}}{2 H_{2}}}$, whereas for the case $q-42 m_{4}>0$ it is even about $X=0$ but with a minimum at the origin and two maxima. Therefore, the magnitudes and the relative signs of the quadrupole and $2^{4}$-pole moments determine the slope of the linear density. Since the LM solution is constructed under the assumption that all the RMM are equally small quantities, we shall consider $\left|\frac{m_{4}}{q}\right|=1,\left(\left|m_{4}\right| \approx|q|\right)$ and hence we have the following density for 
the different cases:

$$
\mu^{L M}(X)=\left\{\begin{array}{c}
\frac{1}{2} \begin{array}{c}
\left.1+\frac{255}{32} q-\frac{615}{8} q X^{2}+\frac{2205}{32} q X^{4}\right) \quad, m_{4} / q=1 \\
\left(q>0 \rightarrow X_{\max }=0, q<0 \rightarrow X_{\min }=0\right)
\end{array} \\
\frac{1}{2}\left(\begin{array}{c}
\left.1-\frac{375}{32} q+\frac{645}{8} q X^{2}-\frac{2205}{32} q X^{4}\right) \quad, m_{4} / q=-1 \\
\left(q>0 \rightarrow X_{\min }=0, q<0 \rightarrow X_{\max }=0\right)
\end{array}\right.
\end{array}\right.
$$

Referring back to point (iii) of the previous section, if we wish to avoid the possibility of a naked singularity we constrain the linear density to be positive definite, and therefore the condition $\mu\left(X_{\min }\right) \geq 0$ must be fulfilled if $X=0$ represents a maximum and $\mu(0) \geq 0$ if $X=0$ represents a minimum of the linear density, i.e. the following relations are found:

$$
\begin{array}{cl}
\mu\left(X_{\text {min }}\right)^{L M} \geq 0 \Leftrightarrow H_{0}-\frac{H_{1}^{2}}{4 H_{2}} \geq 0 & , m_{4} / q=1, q>0 \\
& m_{4} / q=-1, q<0 \\
\mu\left(X_{\text {min }}=0\right)^{L M} \geq 0 \Leftrightarrow H_{0} \geq 0 \quad & , m_{4} / q=1, q<0 \\
& m_{4} / q=-1, q>0
\end{array}
$$

These conditions, (52) and (51), lead to different constraints on the magnitude of the parameters $q$ and $m_{4}$ depending on their relative sign, which can be summarized as follows

$$
\begin{aligned}
& \frac{m_{4}}{q}=1:\left\{\begin{array}{ll}
q>0: & q \leq \frac{1568}{21125} \\
q<0: & |q| \leq \frac{32}{255}
\end{array} \quad \Rightarrow q \in\left[-\frac{32}{255}, \frac{1568}{21125}\right]\right. \\
& \frac{m_{4}}{q}=-1:\left\{\begin{array}{cc}
q>0: & q \leq \frac{32}{375} \\
q<0: & |q| \leq \frac{1568}{18605}
\end{array} \quad \Rightarrow q \in\left[-\frac{1568}{18605}, \frac{32}{375}\right]\right.
\end{aligned}
$$

Or, in other words, if the quadrupole $(q)$ and $2^{4}$-pole $\left(m_{4}\right)$ moments have equal sign regardless of whether the object is flattened or elongated, then the magnitude of both parameters must be restricted to the approximate values $[-0.1255,0.0742]$ in order to conserve a positive definite linear density. In the other cases, $q$ and $m_{4}$ being of different sign, the approximate constraint $[-0.0843,0.0853]$ is required for a well behaved linear density. 
Figure 2 shows schematic representations of the dumbbell (or a bar alone) along with their densities and the masses of the balls for different solutions, starting from the spherically symmetric case (Schwarzschild).

Finally we show the gravitational potential calculated explicitly for this density (49). According to expression (33) and taking into account equations (39), we find

$\Phi=\frac{1}{2} \gamma(\rho, z) \ln \left(\frac{z+M-r_{+}}{z-M-r_{-}}\right)+\frac{M}{2}\left[\beta_{-}(\rho, z) r_{+}-\beta_{+}(\rho, z) r_{-}\right]-\nu\left(\frac{1}{r_{+}}+\frac{1}{r_{-}}\right)$,

where

$$
\begin{aligned}
\gamma(\rho, z) & \equiv H_{0}+H_{1}\left(\frac{2 z^{2}-\rho^{2}}{2 M^{2}}\right)+H_{2}\left[\frac{1}{8 M^{4}}\left(8 z^{4}+3 \rho^{4}-24 \rho^{2} z^{2}\right)\right] \\
\beta_{ \pm}(\rho, z) & \equiv \frac{15}{16} \frac{q}{M^{3}}(3 z \pm M)-\frac{105}{128} \frac{m_{4}}{M^{3}}(95 z \pm 27 M)+ \\
& +\frac{2205}{768} \frac{m_{4}}{M^{5}}\left(50 z^{3}-55 \rho^{2} z \pm 26 M z^{2} \mp 9 M \rho^{2}\right) .
\end{aligned}
$$

$H_{i}$ are the coefficients of the density (49) except for a factor $1 / 2$, and $\nu$ represents the mass (50) of each ball of the dumbbell, which is given by (30) or equivalently (27). It should be noted that this expression (55) leads to the gravitational potential of the M-Q ${ }^{(1)}$ solution for $m_{4}=0$.

\section{Conclusions}

This work is devoted to finding a physical object12 whose Newtonian gravitational potential equals the metric function of some relativistic axially symmetric solutions of the static vacuum equations. Since the static and axisymmetric solutions are defined by means of only one metric function $(\Psi)$, our claim is that the object and its physical features (such as its density) can be used to characterize the Weyl solution by supplying it with an interpretation that generalizes the case of spherical symmetry.

This identification of the solution is, of course, dependent on the system of coordinates but the results obtained here acquire even more interest because

\footnotetext{
${ }^{12}$ The object called a dumbbell and its density have been defined from a singular mass distribution along the $\mathrm{Z}$ axis, but the rod of the dumbbell can also be considered to be a cylinder whose width $\epsilon$ is sufficiently small relative to its length $2 L$ that the ratio $\epsilon / L$ is negligible.
} 


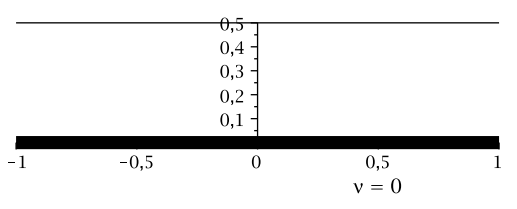

Schwarzschild

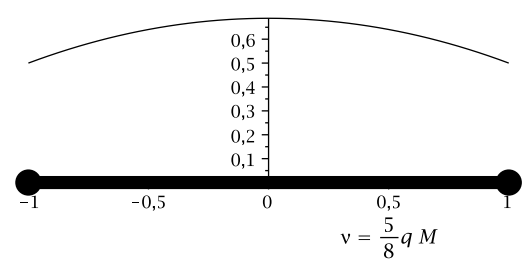

$M Q^{(1)}(q<0)$

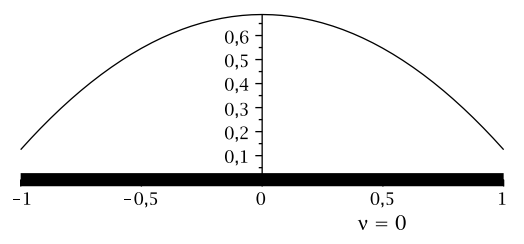

Erez-Rosen $\left(q_{2}<0\right)$

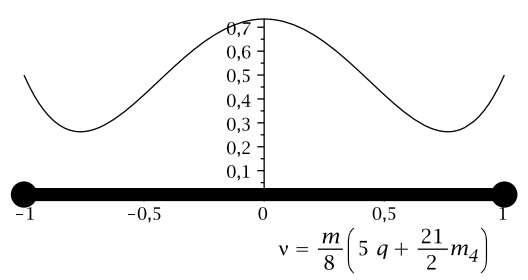

$L M\left(M-Q-2^{4}\right.$ pole $)\left(q<0, m_{4}>0\right)$

Figure 2: The characterization of different Weyl solutions by means of either a bar or a dumbbell. The linear density is represented as well as the mass of the dumbbell balls for those solutions.

the Schwarzschild solution is known to be interpreted, in Weyl coordinates, as a finite bar of constant density. We focus our search on relativistic solutions with a well known physical meaning in the sense of being slight deviations from spherical symmetry. To this end, a new static and axisymmetric solution of the Einstein vacuum equations is presented: the Linearized Multipole (LM) solutions, which represent approximations, linear in the RMM, to the general Pure Multipole solutions, which are defined as those having a finite number of RMM. The particular case of two RMM alone is the M-Q solution [9]. In NG there is no interaction between the multipole moments, and multipole coupling makes no contribution to the gravitational potential. The LM solution is constructed from the contributions of a finite number of RMM 
without any coupling between them. Therefore, the solution can be used to describe very slight deviations from the case of spherical symmetry, due not only to the quadruple moment (the $\mathrm{M}-\mathrm{Q}^{(1)}$ solution is recovered in that case) but also to any other higher RMMs, if they are very small quantities.

If we consider the LM solution with only the first RMM (the monopole), we recover the Schwarzschild solution and consequently the density of the dumbbell bar is constant and its balls vanish. As increasingly higher RMM are considered, then the density of the bar changes in accordance with those RMM and the masses of the balls acquire a non-zero value. We have proved that the object called a dumbbell is able to describe the LM solutions. For instance, the flattened or elongated form of the source of a static and axisymmetric space-time is related to the behaviour of the dumbbell density. We have shown that in this representation the horizon $(x=1)$ of the LM solution is reduced to the region along the $Z$ axis where the rod of the dumbbell is located and the behaviour of the $g_{00}$ component of the metric on that surface can be described by means of the density of the dumbbell. The dumbbell representation allows us to identify the deformations from spherical symmetry due to different RMM in GR by means of the physical characteristics of a Newtonian object.

The definition of the dumbbell and its density allows us to construct an alternative representation of some Weyl solutions: a simple polynomial with $g$ independent terms describes the relativistic solution with $g+1$ multipole moments (starting from the Monopole). The representation of this kind of solution with a finite number of RMM is a clear achievement, and represents an improvement with respect to the Weyl or ERQ representations. Recall that not all Weyl solutions can be identified with the potential of a dumbbell, so the search for an object able to describe other solutions may be a matter of consideration for future work. Moreover, we might consider extended Newtonian objects rather than singular sources to represent the gravitational potential in particular cases. In fact, we should point out that it is possible to obtain general results about the existence of an even density with prescribed moments like those of equation (6):

$$
M_{2 n}=\int_{-1}^{1} z^{2 n} \mu(z) d z=\int_{0}^{1} w^{n}\left[\frac{\mu(\sqrt{w})}{\sqrt{w}}\right] d w,
$$

since Hausdorff [23] proved a set of necessary and sufficient conditions for the existence of a positive function $f$ with prescribed half-range moments $b_{n}$ 
in the sense of equation (57) $b_{n}=\int_{0}^{1} w^{n} f(w) d w$ that involve conditions on the moments (we are referring to the classical problem in analysis called the Hausdorff Moment Problem.)

As a line of enquiry, future work might be devoted to studying what other properties or features of the relativistic solutions can be described in terms of the density of a dumbbell bar. In addition, methods for integrating the equations for the second metric function $\gamma$ in the LM solutions deserve detailed study [24], as does the determination of circular orbits for a test particle around a source with this kind of vacuum metric.

\section{Appendix}

\subsection{Newtonian multipole moments and gravitational potential associated with Letelier's definition of linear density}

Let us calculate the Newtonian gravitational potential, as well as the multipole moments of a bar with density $\lambda(z)$ (11) and length $2 L$, according to the expressions (5) and (6). On the one hand, the gravitational potential is as follow 13 :

$$
\Phi=\int_{-L}^{L} d z^{\prime} \frac{\lambda\left(z^{\prime}\right)}{r} \sum_{n=0}^{\infty}\left(\frac{z^{\prime}}{r}\right)^{n} P_{n}(\omega)=\int_{-1}^{1} \lambda(L t) \sum_{n=0}^{\infty} t^{n} s^{n+1} P_{n}(\omega) d t
$$

where $r \equiv \sqrt{\rho^{2}+z^{2}}, s \equiv L / r$ and the change of variable $z^{\prime}=L t$ has been performed. If we introduce the density (11) into this expression, then the gravitational potential turns out to be:

$$
\Phi=\frac{1}{2} \sum_{n, k=0}^{\infty} P_{n}(\omega) \int_{-1}^{1} s^{n+1} t^{n} q_{k} P_{k}(t) d t=\sum_{n, k=0, k \leq n}^{\infty} q_{k} s^{n+1} P_{n}(\omega) \frac{C_{n, k}}{2 k+1},
$$

where $C_{n, k}$ are the coefficients appearing in the series expansion of the $n^{\text {th }}$ power of any variable as a linear combination of Legendre polynomials in that variable: $\xi^{n}=\sum_{k=0}^{\infty} C_{n, k} P_{k}(\xi)$. In [9], [4] we explicitly obtained the relation

\footnotetext{
${ }^{13}$ Let us note that $\frac{1}{\sqrt{\rho^{2}+(z-x)^{2}}}=\frac{1}{r} \sum_{n=0}^{\infty}\left(\frac{x}{r}\right)^{n} P_{n}(z / r)$.
} 
between the sets of coefficients associated with both the ERQ (10) and the Weyl (1) representations of the axially symmetric static vacuum solutions:

$$
q_{2 k}=(4 k+1) \sum_{j=0}^{k} \frac{L_{2 k, 2 j}}{-M^{2 j+1}} a_{2 j} \quad, \quad q_{2 k+1}=0
$$

where $L_{2 k, 2 j}$ is the coefficient multiplying the $2 j$ power of the variable $\xi$ in the Legendre polynomial $P_{2 k}(\xi)$. Hence, the Newtonian potential (59) transforms into the following expression after taking (60) into account:

$$
\begin{aligned}
\Phi & =-\sum_{n=0}^{\infty} \sum_{k=0}^{n} s^{2 n+1} P_{2 n}(\omega) C_{2 n, 2 k} \sum_{j=0}^{k} L_{2 k, 2 j} \frac{a_{2 j}}{M^{2 j+1}}= \\
& -\sum_{n=0}^{\infty} s^{2 n+1} P_{2 n}(\omega) \sum_{j=0}^{n} \frac{a_{2 j}}{M^{2 j+1}} \sum_{k=j}^{n} C_{2 n, 2 k} L_{2 k, 2 j}
\end{aligned}
$$

and so we can finally conclude that 14 the Newtonian gravitational potential $\Phi$ is equal to the metric function $\Psi$ of the Weyl family of solutions (1):

$$
\Phi=\Psi=-\sum_{n=0}^{\infty} r^{-(2 n+1)} P_{2 n}(\omega) a_{2 n}
$$

On the other hand, in terms of the multipole moments of the bar, we can write, according to (6), the following expression:

$$
M_{2 n}^{N G}=\left(\pi \epsilon^{2}\right) \int_{-1}^{1} L^{2 n+1} t^{2 n} \lambda(L t) d t,
$$

and the introduction of the density $\lambda(z)$ (11) into the integral leads to

$$
M_{2 n}^{N G}=\frac{1}{2}\left(\pi \epsilon^{2}\right) L^{2 n+1} \sum_{k=0}^{\infty} q_{k} \int_{-1}^{1} t^{2 n} P_{k}(t) d t,
$$

\footnotetext{
${ }^{14}$ Let us note that we take $L=M$ and we have used the relation (see [4 for details): $\sum_{k=j}^{n} C_{2 n, 2 k} L_{2 k, 2 j}=\delta_{j n}$.
} 
and we can conclude with the following identity 15 :

$$
M_{2 n}^{N G}=\left(\pi \epsilon^{2}\right) L^{2 n+1} \sum_{j=0}^{n} q_{2 j} \frac{C_{2 n, 2 j}}{4 j+1}=-a_{2 n} .
$$

In other words, we recover the inverse relation of (60) (see [7] for details) existing between the sets of coefficients associated with both the ERQ (10) and the Weyl (1) representations of the axially symmetric static vacuum solutions, and hence we can say that the Newtonian multipole moments of the bar with density given by $\lambda(z)$ (11) are simply the coefficients $a_{2 n}$ of the Weyl family of solutions.

\section{Acknowledgments}

This work was partially supported by the Spanish Ministerio de Ciencia e Innovación under Research Project No. FIS2012-30926, and the Consejería de Educación of the Junta de Castilla y León under the Research Project Grupo de Excelencia GR234.

\section{References}

[1] H. Weyl Ann. Phys. (Leipzig) 54, 117 (1917).

[2] Bäckdahl, T., Herberthson, M., (2005) Class. Quantum Grav. 22, 3585. Herberthson, M., (2004) Class. Quantum Grav. 21, 5121.

Bäckdahl, T., Herberthson, M., (2006) Class. Quantum Grav. 23, 5997.

[3] H. Quevedo Phys. Rev. 33, 334 (1986).

[4] Hernández-Pastora, J.L.,Ph.D. Relativistic gravitational fields close to Schwarzschild solution, Universidad de Salamanca. (1996). (http://campus.usal.es/gmaafig/documentacion/TESIS_JLHP)

[5] J. L. Hernández-Pastora Class. Quantum Grav. 27, 045006 (2010).

\footnotetext{
${ }^{15}$ We have used the following relation: $\int_{-1}^{1} t^{2 n} P_{k}(t) d t=\frac{2 C_{2 n, 2 j}}{4 j+1}$ iff $k=2 j$ (even) with $C_{2 n, 2 j}=0$ for $j>n$. In the case of an odd $k(k=2 j+1)$ the integral is zero.
} 
[6] Letelier, P.S. Class. Quantum Grav. 16, 1207-1213 (1999).

[7] Hernández-Pastora, J.L., Martín, J. (1993) Class. Quantum Grav. 10, 2581.

[8] G. Erez and N. Rosen Bull. Res. Council Israel 8F, 47, (1959).

[9] Hernández-Pastora, J.L., Martín, J. (1994) Gen. Rel. and Grav. 26, 877.

[10] Hernández-Pastora. (2006) Gen. Rel. and Grav. 38, 871. HernándezPastora, J.L., Martín, J and E. Ruiz (1998) Gen. Rel. and Grav. 30, 999.

[11] Bäckdahl, T., Herberthson, M., (2005) Class. Quantum Grav. 22, 1607.

[12] Curzon, H.E.J. Proc. London Math. Soc. 32, 477. (1924).

[13] Abramowitz,M., Stegun, I.A., Handbook of mathematical functions. Dover Publications, Inc. New York. (1972)

[14] Fodor, G., Hoenselaers, C. and Perjés Z., J. Math. Phys., 30, 2252, (1989).

[15] Geroch, R., J. Math. Phys. 11, 1955 (1970) Geroch, R., J. Math. Phys. 11, $2580(1970)$

[16] Thorne, K.S., Rev. Mod. Phys. 52, 299 (1980)

[17] Herrera, L. and Hernández-Pastora Journal of Math. Phys. 41, 7544. (2000)

[18] Hernández-Pastora, J.L, and Ospino, J. Phys. Rev. D 82, 104001 (14 pages) (2010).

L. Herrera, (2005) Found. Phys. Lett. 18, 21-36.

L. Herrera, J. Carot, N. Bolivar and E. Lazo, Int.J.Theor.Phys. 48, 3537-3546, (2009)

[19] W. Israel Phys. Rev. 164, 1776 (1967).

[20] Stephani H. et al, Exact Solutions to Einstein's Field Equations. Cambrigde University Press. 2nd Edition, (2003) 
[21] H. Quevedo, Fortschritte der Physik 38, 733-840 (1990).

[22] Ts.I. Gutsunaev, N.Sh. Hassan and S.L. Elsgolts Gravitation $\& 6$ Cosmology 8, 249-260 (2002).

[23] Hausdorff, F. Summationsmethoden und Momentfolgen. I. Mathematische Zeitschrift 9, 74-109, (1921). Hausdorff, F. Summationsmethoden und Momentfolgen. II. Mathematische Zeitschrift 9, 280-299, (1921).

[24] A. F. Teixeira Progr. Theor. Phys. 60, 163 (1978). 